\title{
Erratum to: Decreased expression of MEG3 contributes to retinoblastoma progression and affects retinoblastoma cell growth by regulating the activity of $W n t / \beta$-catenin pathway
}

Yali Gao ${ }^{1,2} \cdot$ Xiaohe $\mathrm{Lu}^{1}$

Published online: 27 January 2016

C) International Society of Oncology and BioMarkers (ISOBM) 2016

Erratum to: Tumor Biol.

DOI 10.1007/s13277-015-4564-y

The original version of this article unfortunately contained an error in the article category section.

The correct article category of this article is ORIGINAL ARTICLE.

The online version of the original article can be found at http://dx.doi.org/ 10.1007/s13277-015-4564-y.

Xiaohe Lu

luxhgz@126.com

Department of Ophthalmology, Zhujiang Hospital of Southern Medical University, Guangzhou 510282, People's Republic of China

2 Department of Ophthalmology, Shenzhen People's Hospital,

Shenzhen 518020, People's Republic of China 